\section{Ist das Erkrankungsrisiko durch Asbest quantifizierbar?}

\author{
Es besteht ein Zusammenhang zwischen der kumulativen, \\ lebenslangen Asbestbelastung und dem Risiko ein \\ Pleuramesotheliom zu entwickeln. Die dosisabhängige \\ Assoziation scheint jedoch durch geschlechtsspezifische \\ Abweichungen modifiziert zu sein. A. Lacourt et al. haben das \\ Risiko der Asbestexposition in Frankreich genauer analysiert.
}

Thorax 2014; 69: 532-539

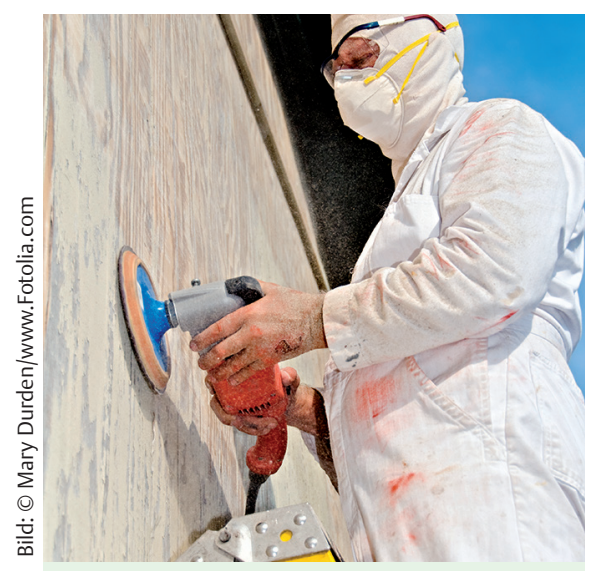

Ob auf der Arbeit oder im Privaten - bei unsachgemäßem Umgang mit asbesthaltigen Materialien steigt das Lungenkrebsrisiko.

Der kausale Zusammenhang zwischen der Asbestexposition und der Ausbildung eines Mesothelioms gilt als gesichert. Noch offen ist die Frage, in welchem Umfang dieser Zusammenhang dosisabhängig ist und wie berufliche und private Expositionen hierbei zu gewichten sind. Auch der geschlechtsspezifische Aspekt bei der Risikobewertung war bisher nicht hinreichend beleuchtet.

\section{Berufliche und private Exposition $\nabla$}

Grundlage der Studie bildeten die epidemiologischen Daten von 437 Patienten, bei denen zwischen 1998 und 2002 ein Pleuramesotheliom diagnostiziert worden war. Eine adjustierte Gruppe aus der gesunden Bevölkerung $(n=874)$ diente als Kontrolle. Die differenzierte Asbestanamnese erfasste sowohl die berufliche als auch die private Belastung (z.B. häusliche Baumaßnahmen). Der Cumulative Expo- sure Index (CEI) ermöglicht es, die Asbestbelastung kumulativ zu erfassen. Er erfasst sowohl Expositionsfrequenz als auch -intensität und erlaubt eine grobe Quantifizierung. Hieraus lässt sich dann das Lebenszeitrisiko errechnen. Die betroffenen Männer ( $n=362)$ waren überwiegend im technisch-handwerklichen Bereich tätig. Die erfassten Frauen dagegen eher im Verwaltungsbereich $(n=75)$.

Die Wissenschaftler fanden einen klaren Zusammenhang zwischen der errechneten Dosisbelastung und dem Mesotheliomrisiko. Geschlechtsunabhängig war bei geringer Belastung das Risiko 4-fach erhöht (Odds Ratio [OR] 4,0). Bei starker Belastung stieg es um den Faktor 67 (OR 67,0). Die berufliche Belastung konnte beim Mann $83 \%$, bei den Frauen dagegen nur $42 \%$ der Mesotheliomfälle erklären. Auffallend war, dass bei Frauen 35\% der Erkrankungen weder durch private noch durch berufliche Exposition erklärt werden konnten. Bei den Männern betrug diese Rate nur $13 \%$.

\section{Fazit}

Bei Verdacht auf Pleuramesotheliom muss die kumulative Asbestexposition als entscheidender Risikofaktor beachtet werden. Sowohl die berufliche als auch die private Anamnese sind zu erheben. Offen bleibt dabei die Frage, warum bei Frauen über ein Drittel der Fälle nicht durch die bisher etablierten Methoden der anamnestischen Risikoerfassung erklärt werden können. Hier offenbaren sich nach Meinung der Autoren erhebliche methodische Defizite.

\section{Dr. Horst Gross, Berlin}

Epidemiologie

\section{Antibiotikavergabe im EU-Vergleich}

Eine Studie im EU-Projekt Aritmo untersuchte die Vergabe von Antibiotika an Kinder und Jugendliche in 5 europäischen Ländern. Es zeigte sich: Am häufigsten werden Antibiotika für Kinder und Jugendliche in Italien verschrieben - gefolgt von Deutschland, England und Dänemark. In den Niederlanden ist die Verschreibungsrate mit Abstand am geringsten - sie beträgt ein Drittel von der in Italien. In allen 5 Ländern erhalten die unter 4-Jährigen am häufigsten Antibiotika. Dies zeigt eine Studie im Zeitraum von 2005 bis 2008, die Daten von 23 Mio. Kindern und Jugendlichen ausgewertet hat. Prof. Edeltraut Garbe, Bremen, ist Leitautorin der Studie, die im September im Journal BMC Pediatrics erschienen ist.

Antibiotika werden häufig verschrieben, zu häufig - denn weltweit nehmen resistente Bakterienstämme zu, durch die Antibiotika ihre Wirkung verlieren. Um gegenzusteuern, ist es grundlegend zu verstehen, wie Antibiotika verordnet werden. In der publizierten Studie, die im EU-Projekt Aritmo über Nebenwirkungen verschiedener Medikamentengruppen erfolgte, haben Forscher die Häufigkeit, aber auch die Art des verschriebenen Antibiotikums bei verschiedenen Altersgruppen von 0 bis 18 Jahren beleuchtet.

Die Forscher vermuten, dass die Unterschiede nicht durch verschiedene Infektionshäufigkeiten in den Ländern, sondern durch länderspezifisches Verschreibungsverhalten verursacht werden. Es ist anzunehmen, dass Antibiotika nicht nur gegen bakterielle, sondern auch gegen virale Erkrankungen verordnet werden. Dies wird von der Beobachtung gestützt, dass die Verordnung von Antibiotika jahreszeitliche Spitzen hat. Am höchsten ist sie im Winter, insbesondere in den Ländern mit einer hohen Verschreibungsrate. Dies spricht dafür, dass Antibiotika auch gegen virale Infektionen eingesetzt und somit falsch angewendet werden. Hauptsächlich die viralen Erkrankungen steigen im Winter an, nicht die bakteriellen.

Nach einer Mitteilung des Leibniz-Instituts für Präventionsforschung und Epidemiologie - BIPS, Bremen 